\title{
Éditorial
}

\section{RADIOPROTECTION sur le net}

Pour le président du Comité de rédaction comme pour l'éditeur, connaître nos lecteurs est un souci permanent. Ainsi, grâce aux statistiques de consultation du site, nous pouvons avoir une idée de la façon dont est perçu RADIOPROTECTION aussi bien en France que dans le monde.

Rappelons tout d'abord que, outre les abonnés de la revue, les adhérents de la SFRP ont tous accès aux articles dès leur publication en ligne. Ensuite, tous les articles sont accès libre après une période d'embargo de trois ans et ce jusqu'aux numéros publiés en 1990 (soit 20 ans d'archives de la revue). Quant aux numéros spéciaux, ils sont en accès libre dès leur parution. Enfin, tout le monde peut, gratuitement, recevoir la table des matières par email en s'inscrivant à la rubrique «e-mail alert» du site ou directement sur son nagivateur grâce aux fils RSS (services disponibles sur le site de notre revue, www.radioprotection.org). Grâce à ces services, vous connaitrez le contenu du numéro environ 15 jours avant sa livraison dans vos boîtes à lettre.

Tous les ans nous procédons donc à l'examen des statistiques de téléchargement et pour 2009, l'article le plus téléchargé est Dynamics of Carbon 14 in soils: a review par Christian Tamponnet (Vol. 40, suppl. 1, 465-470), puis celui de Olivier Couasnon et al., Le référentiel règlementaire (Vol. 43, $\mathrm{N}^{\circ}$ 7, 11-33) et, si l'on ne cite que le podium, vient ensuite la rubrique Internet éclaire de décembre 2008 que nous devons comme pour chaque numéro à Sandrine Figueiredo. La liste complète des 20 articles les plus téléchargés figure sur le site de la revue. Le Comité félicite ces auteurs et les remercie de leur collaboration. De plus vous avez remarqué que deux sont membres du Comité de rédaction de Radioprotection.

L'examen de cette liste montre bien ce que les lecteurs attendent de nous une revue professionnelle décrivant les multiples facettes de la radioprotection où les aspects réglementaires figurent en bonne place. Il montre également le succès de nos numéros spéciaux, souligné encore plus clairement si l'on se borne aux articles publiés uniquement en 2009. Ceci devrait de facto encourager les organisateurs des journées SFRP à convaincre les auteurs de présentations orales à les publier dans notre journal.

La mise en ligne récente des archives de la revue depuis 1990 est un succès ; 6252 chargements pour les années 1990-1996 et sans surprise c'est un article publié en 1996 sur le radon, toujours d'actualité, qui est le plus téléchargé (Le radon-222 et ses descendants à vie courte dans l'environnement atmosphérique : origine et méthodes de mesure par J. Charuau et al., Vol. 31, $\mathrm{N}^{\circ} 3,371-388$ ).

DOI: $10.1051 /$ radiopro/2010004

RADIOPROTECTION - VOL. 45 - N 1 (2010) 
ÉDITORIAL

Ces statistiques, reflets de l'année 2009 vont, nous l'espérons, changer. En effet, nous sommes maintenant référencés sur la base ISI / Web of Science : beaucoup de scientifiques utilisant cette base pour faire leur bibliographie, cela devrait amener à notre revue de nouveaux lecteurs de tous horizons.

Pour conclure, notre message reste le même : envoyez nous des articles, en français ou en anglais, sans oublier de consulter les instructions aux auteurs où figure maintenant un programme pour faciliter la rédaction des références (endnote). Orateurs de nos journées soyez conscients qu'une concrétisation de vos présentations dans la revue multipliera leur impact. Mais soyez en sûr, nous ne baisserons pas la garde au niveau de la qualité, si 2009 a été une année record en termes d'articles reçus, près de $30 \%$ ont été refusés, nos évaluateurs veillent..., qu'ils en soient remerciés.

À vos plumes pour une année 2010 encore meilleure

H. Métivier

Président du Comité de rédaction

A. Henri

EDP Sciences 